\title{
The potential use of a three dimensional cell culture of spheroids in the study of various therapeutic approaches
}

Volume 3 Issue 4 - 2016

\begin{abstract}
Abbreviations: FDA, food and drug administration; EDM, enteroid-derived epithelial monolayers; CF, cystic fibrosis; CFTR, cystic fibrosis transmembrane conducting regulator; MCTS, multicellular tumor spheroid
\end{abstract}

\section{Mini review}

With the advancement of biomedical science, the number of clinical trials and drug testing is accelerating but still ninety percent of experimental drugs fail in clinical studies. Some of the major reasons behind the failure are the pathophysiological differences between human diseases and the animal model/human cell lines tested. As a result, there has been a $40 \%$ decrease in the number of drugs the Food and Drug Administration (FDA) has approved since 2005. ${ }^{1}$ Traditional two dimensional models of cell culture have proven to have limitations in how accurately they mimic in vivo circumstances of cells. Progress in the study of human stem cell technology has allowed for the development of three dimensional cell cultures that may more accurately reflect the pathophysiology of human diseases. Three dimensional cell cultures allow for increased cell-to-cell and extracellular matrix interactions and are closer in structure and architecture to in vivo organs and tissues. The use of spheroids, a three dimensional cell culture method, has allowed for greater understanding and advances in cancer cell interactions and testing toxicity, drugs, and therapeutic methods. Spheroid use in the study of gastrointestinal tract diseases, cystic fibrosis research, and cancer research and therapy will be discussed in this mini-review.

Spheroids organize to make a three-dimensional culture of cells. The specific term for cell cultures of human adult stem cells from the gastrointestinal tract is an enteroid model. The mucosal epithelial cells maintain the integrity to protect host from toxic compound and pathogens. So far, our knowledge was limited with the established epithelial cell line model that does not correlate the in vivo situation of human diseases. Utilizing the progress of stem cell research, Sato, et al. ${ }^{2}$ first developed a defined condition for the growth and expansion of intestinal stem cells as 3D intestinal organoids or enteroids. ${ }^{2,3}$ Enteroids mimic in vivo situation with four different cell types: epithelial, goblet, Paneth cells and enterocytes. The 3-dimensional spheres with 200-400 epithelial cells could be dissociated and plated onto transwells as monolayers known as the Enteroid-Derived epithelial Monolayers (EDM). The EDM maintains the similar architecture with same percentage of cell population that exist in vivo ${ }^{1,4}$ On top of that, the polarization of the epithelium in the EDM can access the apical and basolateral sides separately. ${ }^{1,4}$

Enteroids are formed under modified conditions that enhance the viability of the cells. Themedia used for the growth of the gastrointestinal stem cells is made from a supportive cell line called L-WRN. L-WRN secretes WNT, R-spondin, and noggin, which are three factors that are needed and sufficient for the propagation and maintenance of

\author{
Soumita Das, Ayse Z Sahan, Eileen Lim, \\ Katherine Suarez \\ Department of Pathology, University of California, USA
}

Correspondence: Soumita Das, Department of Pathology, University of California, USA, Tel 858-822-5523, Emailsodas@ucsd.edu

Received: July 0I, 2016 | Published: June 05, 2016

intestinal epithelial cells. These factors are necessary to prevent the stem cells from differentiating into different epithelial cells types such as Goblet cells, Paneth cells, enterocytes, enteroendocrine cells, M cells, and tuft cells. The secretions of L-WRN prevent differentiation and are necessary for growing cells for maintenance. ${ }^{2,5}$ Furthermore, to maximize the early growth of developing primary enteroids, the media is supplemented with an inhibitor for Rho-associated protein kinase and an inhibitor for TGF- $\beta$ type I receptor, which allows the enteroids to propagate from different sites using the same media. By properly preserving gastrointestinal stem cells using the technique of growing them as spheroids or enteroids, there can be maintenance of a culture of cells that can be later used for studies involving infections. With infections, there is a change in media type where there will no longer be L-WRN secretions that allow the stem cells to differentiate. Studies of the effects of infections on the differentiated cell types can be observed. For maintenance, the use of L-WRN as conditioned media provides proteins that are relatively intact and higher titer than reconstituted media that has purified proteins. ${ }^{6}$ However, the potential disadvantage is that the host cells could possibly secrete undesired factors if there is an exhaustion of media. As a result, there has to be careful timing in harvesting the cells or replacing the culture with fresh media. The protocol for maintaining spheroids has been refined in order to allow for the growth of a sustainable culture of spheroids that can be utilized for various experimental purposes.

Maintenance of spheroids is a demanding task as the media preparation and maintenance conditions are very specific. However, spheroids or organoids are useful for applications in many areas of disease research, especially in the field of personalized disease therapy. Spheroids are considered especially promising for Cystic Fibrosis (CF) research as a human model system capable of facilitating a personalized approach to treatment. Caused by mutations in the cystic fibrosis transmembrane conducting regulator (CFTR) gene, CF is characterized by the impairment of chloride transporters, causing an excess of thick mucus that contributes to chronic or recurrent infections that cause death by eventual lung failure. Mutations in the 
CFTR gene can vary greatly between individuals with $\mathrm{CF}$, leading to difficulty in employing a general model of treatment. While animal models such as mice have been used in research, they are limited by their insufficient resemblance to the phenotypes expressed in humans. Interestingly, there has been progress in studying CFTR function in intestinal epithelial organoids. Epithelial organoids are normally characterized by a large amount of swelling because the apical surface epithelial cells secrete fluid into the sphere where it cannot escape. Isolated from $\mathrm{CF}$ patients, the organoids are induced to swell by using forskolin, a compound that increases the amount of intracellular cAMP, which causes CFTR activation. When treated with drugs to correct CFTR function, the CF intestinal organoids showed more swelling, indicating better CFTR function. This more personalized approach to $\mathrm{CF}$ treatment is also promising in the field of gene therapy through functional gene repair utilizing the CRISPR/Cas9 system.?

Another promising area of spheroid application is cancer research and therapy. Spheroids are increasingly being used to track tumor growth patterns as well as assess tumor responses to therapeutics. To this purpose, research on spheroids in relation to cancer is focused on the mechanisms of proliferation, invasion, and metastasis. Spheroids used in cancer research are composed of tumor cells that mimic the growth of real tumors in a $3 \mathrm{D}$ structure with specific micro-environmental conditions. ${ }^{8}$ The multicellular tumor spheroid (MCTS) model has been used to stimulate the conditions of about 40 different types of malignant cells as well as a limited number of nonmalignant cells. Differences in the model are generally observed in cell thickness, organization, density, and other physical properties amongst different types of spheroids. Previously, monolayer cultures were used in cancer research. However, the arrangement of cells in a 3D culture such as in the MCTS model has been proven to be more reliable due to its similarities to tumors in vivo by antigen expression, various micro-environmental gradients such as $\mathrm{pH}$ and oxygen, and proliferation. Similarities between the spheroids and their respective original tumors such as growth, heterogeneity of cells, and characteristics of differentiation support the usefulness of spheroids in cancer research. The MCTS model has allowed for many discoveries on cancer proliferation, invasion, and metastasis through the use of the MCTS model. ${ }^{9}$ For instance, many studies have discovered that specific portions of spheroids have chronically hypoxic cells, which are immune to almost all forms of therapy. These hypoxic cells in tumors in vivo may be the reason for the failure of cancer treatments in certain cases. New techniques within the MCTS model are being developed that contribute much to our understanding of tumors. ${ }^{10} \mathrm{~A}$ recently developed method of co-culturing called confrontation, in which malignant cells are cultured with nonmalignant cells within a spheroid, allows for the study of invasion. ${ }^{11}$ And finally, tumor spheroids are useful for testing the practicality of various therapeutics including radiation, chemotherapy, and immunotherapy. Researchers have discovered that spheroids exposed to radiation are more resistant to therapy than monolayers due to the presence of hypoxic cells similar to those in tumors in vivo. ${ }^{8}$ In the future, spheroids may be grown from patient tumor samples and used to test the efficiency and use of different cancer treatment therapies as well as to discover more about the proliferation, invasion, and metastasis of malignant cells.

Switching from a two dimensional cell culture model to the three dimensional cell culture of the spheroid method can allow for a more practical and accurate model that mimics the in vivo circumstances of cells. The use of enteroids in gastrointestinal tract disease studies has been developed to allow for optimum growth and maintenance conditions. In the study of Cystic Fibrosis, organoids are used to model personalized approaches to treatment. And lastly, the use of spheroids through the MCTS model in the study of cancer is useful for gaining insight into proliferation, invasion, and metastasis as well as testing and understanding cancer therapy techniques. These methods of spheroid use in impactful fields of study provide insight on the potential advantages of using this method over a two dimensional cell culture method.

\section{Acknowledgments}

None.

\section{Conflicts of Interset}

None.

\section{References}

1. Foulke-Abel J, In J, Kovbasnjuk O, et al. Human enteroids as an exvivo model of host-pathogen interactions in the gastrointestinal tract. Exp Biol Med (Maywood). 2014;239(9):1124-1134.

2. Sato T, Vries RG, Snippert HJ, et al Single Lgr5 stem cells build crypt-villus structures in vitro without a mesenchymal niche. Nature. 2004;459(7244):262-265.

3. Stelzner M, Helmrath M, Dunn JC, et al. A nomenclature for intestinal in vitro cultures. Am J Physiol Gastrointest Liver Physiol. 2003;302(12):G1359-G1363.

4. Davies JM, Santaolalla R, Abreu MT. Use of Cancer Stem Cells to Investigate the Pathogenesis of Colitis-associated Cancer. Inflamm Bowel Dis. 2016;22(4):976-983.

5. Sato T, Stange DE, Ferrante M, et al. Long-term expansion of epithelial organoids from human colon, adenoma, adenocarcinoma, and Barrett's epithelium. Gastroenterology. 2011;141(5):1762-1772.

6. Miyoshi $\mathrm{H}$, Stappenbeck TS. In vitro expansion and genetic modification of gastrointestinal stem cells in spheroid culture. Nat Protoc. 2013;8(12):2471-2482.

7. Mou H, Brazauskas K, Rajagopal J. Personalized medicine for cystic fibrosis: establishing human model systems. Pediatr Pulmonol. 2013;50(Suppl 40):S14-S23.

8. Sutherland RM. Cell and environment interactions in tumor microregions: the multicell spheroid model. Science. 1988;240(4849):177-184.

9. Ho WY, Yeap SK, Ho CL, et al. Development of multicellular tumor spheroid (MCTS) culture from breast cancer cell and a high throughput screening method using the MTT assay. PLoS One. 2001;7(9):e44640.

10. Thoma CR, Zimmermann M, Agarkova I, et ak. 3D cell culture systems modeling tumor growth determinants in cancer target discovery. Adv Drug Deliv Rev. 2014;69-70:29-41.

11. Wartenberg M, Dönmez F, Ling FC, et al. Tumor-induced angiogenesis studied in confrontation cultures of multicellular tumor spheroids and embryoid bodies grown from pluripotent embryonic stem cells. FASEB J. 2001;15(6):995-1005. 